\title{
PATHOLOGICAL AND IMMUNOLOGICAL STUDY OF AN IN OVO COMPLEX VACCINE AGAINST INFECTIOUS BURSAL DISEASE
}

\author{
Mária Kelemen $^{1 *}$, Katalin ForgÁCH ${ }^{1}$, Judit IVÁN ${ }^{2}$, V. PAlyA ${ }^{1}$, T. SÜVEGES ${ }^{1}$, B. Tóth ${ }^{1}$ \\ and J. MÉSZÁROS ${ }^{2}$ \\ ${ }^{1}$ CEVA-PHYLAXIA Veterinary Biologicals Co. Ltd., H-1107 Budapest, Szállás u. 5, \\ Hungary; ${ }^{2}$ Veterinary Medical Research Institute of the Hungarian Academy of Sciences, \\ H-1581 Budapest, P.O. Box 18, Hungary
}

(Received June 30, 2000; accepted October 17, 2000)

\begin{abstract}
The appearance of very virulent strains of infectious bursal disease (IBD) virus at the end of the 1980s made it necessary to develop more effective immunization procedures. To facilitate this, the immunogenicity and the immunosuppressive effect of a mild (G-87), an intermediate (LIBD) and an intermediate-plus (IBDV 2512) IBDV strain were tested after the in ovo inoculation of 18-day-old SPF and broiler chicken embryos. It was established that no noteworthy difference existed between the immunized and the control embryos in hatching rate and hatching weight. The higher the virulence of the vaccine virus strain, the more severe damage it caused to the lymphocytes of the bursa of Fabricius. In SPF chickens, the haemagglutination inhibition (HI) titres induced by a Newcastle disease (ND) vaccine administered at day old decreased in inverse ratio to the virulence of the IBD vaccine strain, while in broiler chickens this was not observed. Despite the decrease of the HI titre, the level of protection did not decline, or did so only after the use of the 'hot' strain. SPF chickens immunized in ovo with a complex vaccine prepared from strain IBDV 2512 and IBD antibody showed the same protection against Newcastle disease as the broilers. In broiler chicken embryos immunized in ovo, only strain IBDV 2512 induced antibody production, and such chickens were protected against IBD at 3 weeks of age. The complex vaccine administered in ovo has been used successfully at farm hatcheries as well.
\end{abstract}

Key words: Infectious bursal disease virus, in ovo, immunosuppressive effect, SPF embryos, broiler embryos, IBDV strains G-87, LIBD, IBDV 2512

The very virulent infectious bursal disease virus (IBDV) strains that emerged at the end of the 1980s (van den Berg et al., 1991) have called for the development of more effective vaccines (Lütticken, 1997). These efforts have been two-pronged: firstly, they were aimed at the establishment of protection as early in life as possible, since on farms infected with virulent strains IBD tended to occur at an ever earlier age (van den Berg et al., 1991); secondly, attention be-

*E-mail: maria.kelemen@cevavet.com; Fax: +36 (1) 260-3889 
came focused on the use of vaccine virus strains of increased virulence to improve the effectiveness of immunization. However, in the absence of adequate antibodies such strains of increased virulence may severely damage the bursa of Fabricius. In an attempt to prevent this latter effect, the optimum time of immunization was calculated from the initial level of maternal antibodies. Since, however, the antibody levels tend to vary widely within a flock, vaccine strains of higher virulence (e.g. the intermediate-plus vaccine strains) may cause immunosuppression in chickens having a lower level of maternal antibodies.

The earliest possible development of immunity can be expected of the in ovo vaccination of 18-day-old embryos, while the potential side effects of the highly immunogenic intermediate-plus strains can be prevented by homologous antibody added to the vaccine. Thus, the in ovo administered so-called complex vaccine (Whitfill et al., 1995) promises to be the ideal solution. The use of this immunization method is spreading. Therefore, in this work wide-ranging pathological and immunological studies were carried out with the available vaccine strains in order to select the strain optimal for in ovo immunization.

\section{Materials and methods}

Chickens. In some of the experiments SPF chickens (SPAFAS) were used. The conventional broiler chickens were Ross (He-Ross, Hernád hatchery, Hungary) or Cobbs (Gallus Ltd., Devecser hatchery, Hungary) hybrids. The broiler parent flock was immunized against infectious bronchitis (IB), IBD and Newcastle disease (ND) first with live virus and subsequently, before the laying season, with inactivated vaccine.

Virus strains, immunization. Eighteen-day-old embryos were vaccinated against IBD with the live IBDV strains of CEVA-PHYLAXIA Co. Ltd.: G-87 (mild, CEVAC BURSA L), LIBD (intermediate, CEVAC GUMBO L), and IBDV 2512 (intermediate-plus, CEVAC IBD L) administered in a dose of $10^{2.7-3.0}$ $\mathrm{EID}_{50}, 10^{3.0-3.3} \mathrm{TCID}_{50}$ and $10^{1.8-2.3} \mathrm{EID}_{50}$ virus per embryo, respectively. The embryos were injected with a 1.25-inch-long, 22-gauge needle. Fourteen-day-old chickens were immunized via the drinking water with a $10^{2.0}$ EID $_{50}$ dose of CEVAC IBD L (intermediate-plus) vaccine, after 2-hour deprival of drinking water, administered in $20 \mathrm{ml}$ of drinking water per chicken.

One dose of the complex vaccine (CEVAC TRANSMUNE IBD) contained $10^{2.0} \mathrm{EID}_{50}$ of IBDV 2512 virus and 20 units of immune serum produced against it in SPF chickens. The dose of the immune serum was determined according to the method of Haddad et al. (1997). In the laboratory, the vaccine was administered to 18-day-old embryos as described above, while during the field trial it was given at the hatchery with the Inovoject apparatus (Embrex), in a volume of $0.05 \mathrm{ml}$. 
In the laboratory, day-old chicks were immunized against Newcastle disease with $10^{6.3}$ EID $_{50}$ apathogenic virus (Vitapest, CEVA-PHYLAXIA Co. Ltd.) by the intraconjunctival route (eye drop), in a fluid volume of $2 \times 0.03 \mathrm{ml}$. At the hatchery, that vaccine was administered either by aerosol in a dose of $10^{7.3} \mathrm{EID}_{50} / \mathrm{m}^{3}$ for an exposure time of $15 \mathrm{~min}$ (Mészáros et al., 1992) or it was given in spray form in a virus dose of $10^{8.3} \operatorname{EID}_{50}$ for 100 chicks, using a vaccinator of closed system ('vaccination tunnel').

Determination of the degree of embryo damage. The hatching rate and hatching weight of the in ovo vaccinated embryos and, at times specified for the different experiments, the B:B ratio, B:B index and the histological score indicating the degree of damage of the bursa of Fabricius (Süveges, 1998) were determined.

Efficacy tests. In groups formed from the immunized embryos and from the nonvaccinated control ones the IBD antibody content of individual blood sera was determined by the virus neutralization (VN) test, against 200 TCID $_{50}$ virus, at hatching and then on days $7,14,21,28$ and 35 . The rate of excretion of maternal antibodies and the onset and development of active immune response were studied.

Challenge infection was performed with a very virulent infectious bursal disease virus (vv IBDV) strain designated MOH 94/9, isolated from a Hungarian outbreak, by the injection of $100 \mathrm{LD}_{50}$ virus into the oral cavity in a fluid volume of $0.5 \mathrm{ml} /$ chicken. The challenged animals were observed for 7 days. The average daily weight gain during challenge infection was calculated as the difference of body weight at challenge and that measured 7 days after challenge.

Study of the immunosuppressive effect. The in ovo immunized and control groups were immunized against Newcastle disease at day old in the manner described above, then at 21 days of age the ND antibody levels of the groups were compared, and virulent ND virus challenge was performed. The level of ND antibodies was determined by the HI test against $8 \mathrm{HA}$ units of virus.

The chickens were challenged with $10^{6.0} \mathrm{LD}_{50}$ of virulent NDV strain Herts $33 / 56$, by the intramuscular injection of $0.5 \mathrm{ml}$ fluid. The period of observation was 14 days. Chickens that survived the challenge and did not show signs indicative of ND were considered protected.

The effect of the above three IBD vaccine strains was studied first in SPF chickens and then in broilers. On the basis of the results obtained, the strain that seemed to be the most suitable for use in the complex vaccine was selected. SPF growing chickens were repeatedly immunized with the selected strain to obtain high-titre antibodies. The complex vaccine was prepared by the addition of that antibody, and its pathological and immunological effects were studied in 18-dayold SPF and broiler embryos immunized under field conditions. 


\section{Results and discussion}

The hatching rate, expressed for fertile eggs, of nonvaccinated SPF and broiler embryos and of those inoculated in ovo with PBS or with IBD vaccine virus strains of different virulence did not differ markedly, with the exception of the group immunized with the intermediate IBDV strain LIBD (70 and 80.6\%, respectively). No significant difference was obtained in the weight of the day-old chicks either. In broilers, the most pronounced manifestation of the different virulence of the three vaccine strains was the dissimilarity of the B:B indices and histological scores (Table 1). This indicates that strain IBDV 2512 caused the most severe, though irreversible, damage to the bursa of Fabricius of chickens (Table 2). Despite this fact, when after challenge in weeks 2-5 the histological scores of the bursa of Fabricius of chickens hatched from broiler embryos immunized in ovo with different IBDV strains were compared with those of the controls (nonimmunized but challenged groups), it was found that the 'hotter' the strain used for in ovo immunization, the greater decrease occurred in the score of histological lesions produced by virulent virus challenge. Accordingly, the values of the group immunized with the IBDV 2512 strain were the most favourable (Table 3). This was supported by the finding that in the groups immunized in ovo with the IBDV 2512 strain the average daily weight gain following challenge at 3,4 or 5 weeks was significantly higher already from the 4th week than that of the nonimmunized but challenged controls (Fig. 1).

Comparing some indices of broiler chicken groups vaccinated with the three strains in ovo it was found that the B:B indices decreased whereas the histological scores increased with the virulence increase of the strain (this was the most expressed for the intermediate-plus strain). At the same time, it could be established that, of the broilers having maternal antibodies, only the groups vaccinated with strain IBDV 2512 developed antibodies due to active immunity, by the 4 th week of life (Fig. 2).

In view of these findings, it was important to study how much the (temporary) bursal damage following in ovo immunization was associated with an immunosuppressive effect. From the relevant experiments it was concluded that when chickens hatched from embryos immunized in ovo were vaccinated with a Newcastle disease vaccine at day old, in the 3rd week the HI titres decreased very substantially in the SPF chicks and moderately in the broilers in the case of strain IBDV 2512. Despite this fact, the level of protection was found to be outstandingly high (100\%) in numerous experiments. Only in birds immunized with strain IBDV 2512 was the protection somewhat lower: $81.8 \%$ in SPF chickens but $95.5 \%$ in the broilers, which conforms to the efficacy requirements. 

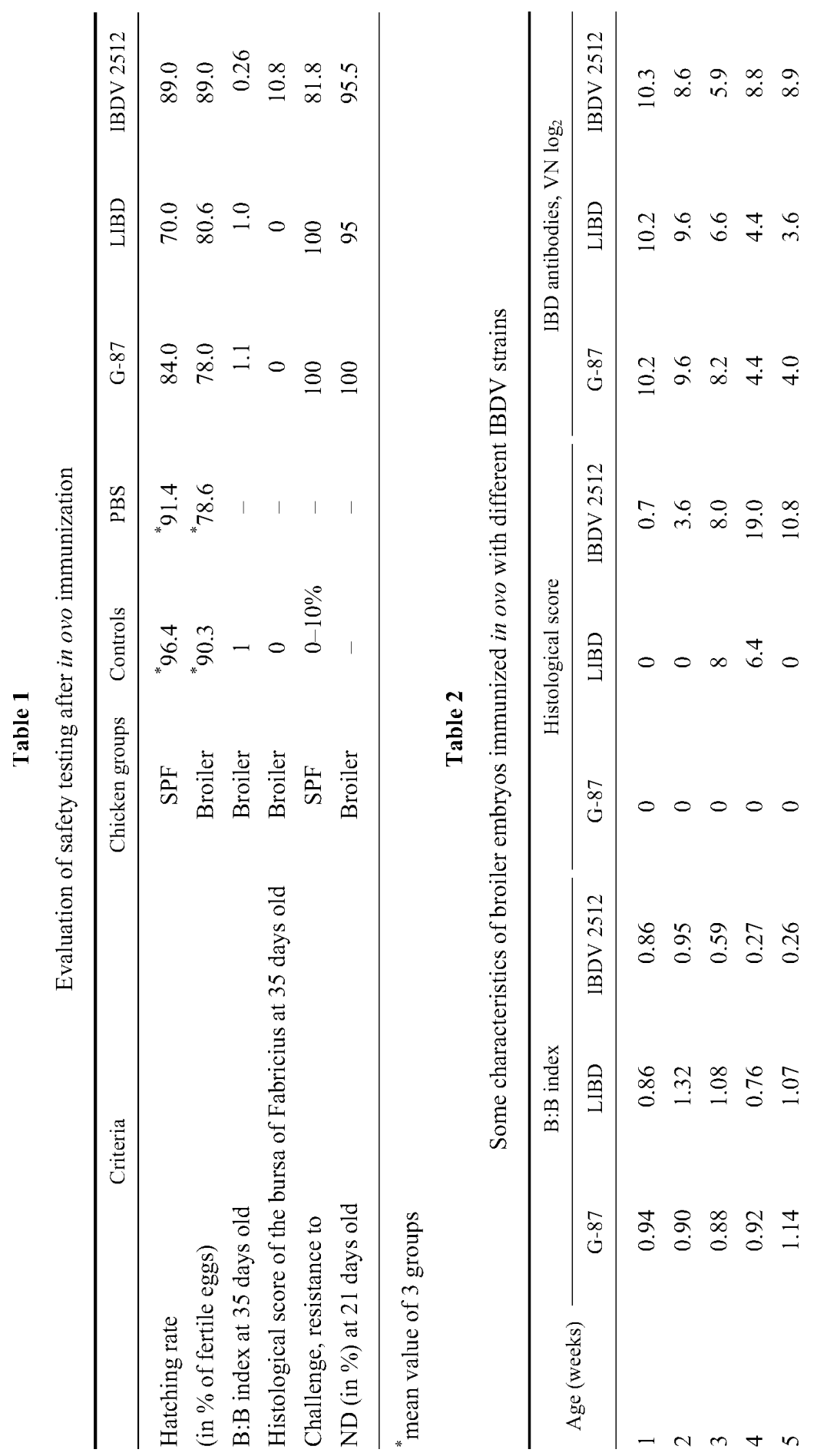
As the level of maternal antibodies varies widely by flock and also by bird, this may pose a risk when applying highly immunogenic, 'hotter' vaccine virus strains. Therefore, a so-called complex vaccine was prepared from a mixture of the intermediate-plus IBDV strain and specific antibodies produced against it. Subsequently the pathological and immunological effects of that vaccine were studied.

Table 3

Histological lesion scores of broilers vaccinated against IBD in ovo

\begin{tabular}{|c|c|c|c|c|c|c|c|c|c|c|c|c|}
\hline \multirow{3}{*}{$\begin{array}{c}\text { Age } \\
\text { (weeks) }\end{array}$} & \multicolumn{12}{|c|}{ Strain } \\
\hline & \multicolumn{4}{|c|}{ G-87 } & \multicolumn{4}{|c|}{ LIBD } & \multicolumn{4}{|c|}{ IBDV 2512} \\
\hline & i. & n.i. & i. + ch. & n.i. + ch. & i. & n.i. & i. + ch. & n.i. + ch. & i. & n.i. & i. + ch. & n.i. + ch. \\
\hline 0 & 0 & 0 & - & - & 0 & 0 & - & - & 0 & 0 & - & - \\
\hline 1 & 0 & 0 & - & - & 0 & 0 & - & - & 0.7 & 0 & - & - \\
\hline 2 & 0 & 0 & 12.1 & 13.5 & 0 & 0 & 12.8 & 16.0 & 3.6 & 0 & 18.6 & 18.6 \\
\hline 3 & 0 & 0 & 19.8 & 20.2 & 8.0 & 0 & 9.3 & 13.0 & 8.0 & 0 & 11.0 & 22.0 \\
\hline 4 & 0 & 0 & 22.8 & 21.3 & 6.4 & 0 & 18.7 & 21.0 & 19.0 & 0 & 10.8 & 22.4 \\
\hline 5 & 0 & 0 & 22.2 & 22.6 & 0 & 0 & 15.2 & 22.6 & 10.8 & 0 & 9.7 & 26.1 \\
\hline
\end{tabular}

i.: immunized at 18 days of incubation; n.i.: non-immunized; i. + ch.: immunized and challenged; n.i.+ch.: non-immunized and challenged

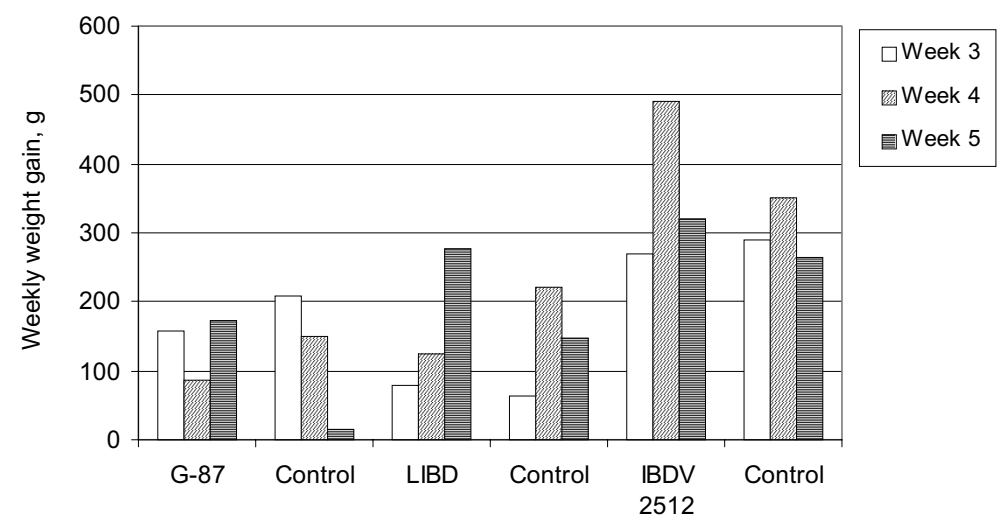

Fig. 1. Weight gain of broilers vaccinated in ovo with different infectious bursal disease vaccines and of control broilers in the week after challenge at 3-5 weeks of age

No marked difference was found between the SPF embryo groups immunized in ovo with the complex vaccine and the control embryo groups in hatching rate ( 97.7 and $90.0 \%$, respectively), and the difference in average body weight was only $1 \mathrm{~g}$, with a very low standard deviation (4.32-3.91) for both values. 
The low histological lesion scores of the bursae of Fabricius found in the weeks after both immunization and virulent virus challenge indicated that only in the 2nd week did the complex vaccine cause distinct histopathological lesions, and the lesions became much milder in the subsequent weeks. It was very favourable that the scores hardly increased after challenge in weeks 4 and 6 with respect to the prechallenge values (Fig. 3).

Studying the spread of vaccine virus within the flock, it was found that SPF contact control birds co-mingled with the in ovo immunized SPF day-old chicks had seroconverted by 2 weeks of age, which indicates that the virus contained in the complex vaccine has the ability to spread. At 7 days old, chicks hatched from SPF embryos immunized in ovo with different virus doses $\left(10^{1.8-2.3}\right)$ showed $6-30 \%$ seropositivity in the $\mathrm{VN}$ test depending on the virus dose, but at 14 days of age all sera gave positive results and contained antibody titres showing minor dose-related differences $(1: 640-1: 870)$.

In the weeks following in ovo immunization with the above complex vaccine at the farm hatchery, after the decline of the maternal antibody levels the IBD VN titres of broilers rose above the value of $9 \log _{2}$ from the 3 rd week, in the same way as in sibling groups immunized at 2 weeks old with an intermediateplus strain (CEVAC IBD-L) administered via the drinking water. After vv IBDV challenge performed in weeks 5 and 6 in chicken groups immunized in ovo and via the drinking water, the highest body weight gain was recorded in the in ovo immunized groups. The body weight gain was slightly lower in the groups immunized via the drinking water at 2 weeks old, and markedly lower in the control groups (Fig. 4).

1. MILD (G-87)

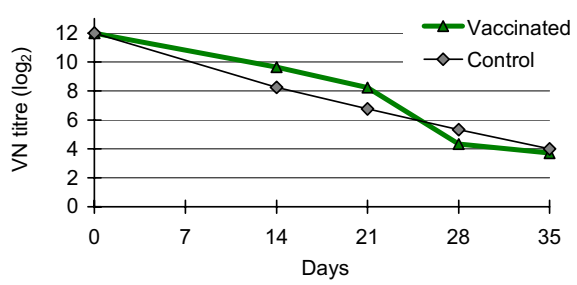

2. INTERMEDIATE (LIBD)

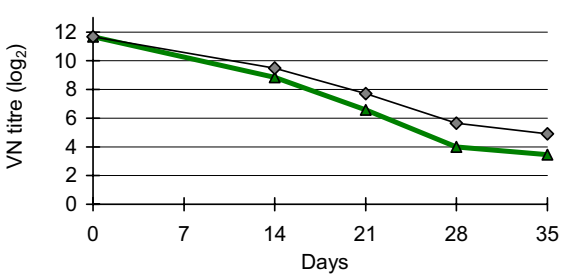

3. INTERMEDIATE PLUS (IBDV 2512)

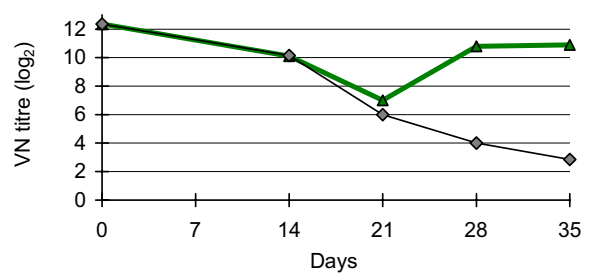

Fig. 2. Immune response of broiler embryos immunized in ovo with IBD vaccines of different virulence groups 


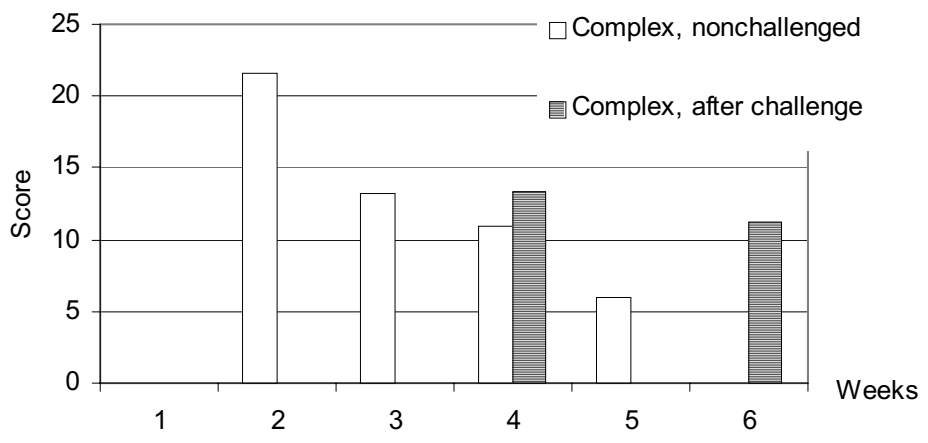

Fig. 3. Weekly histological lesion scores of the bursa of Fabricius of challenged and nonchallenged chickens hatched from SPF embryos immunized with IBD complex vaccine in ovo

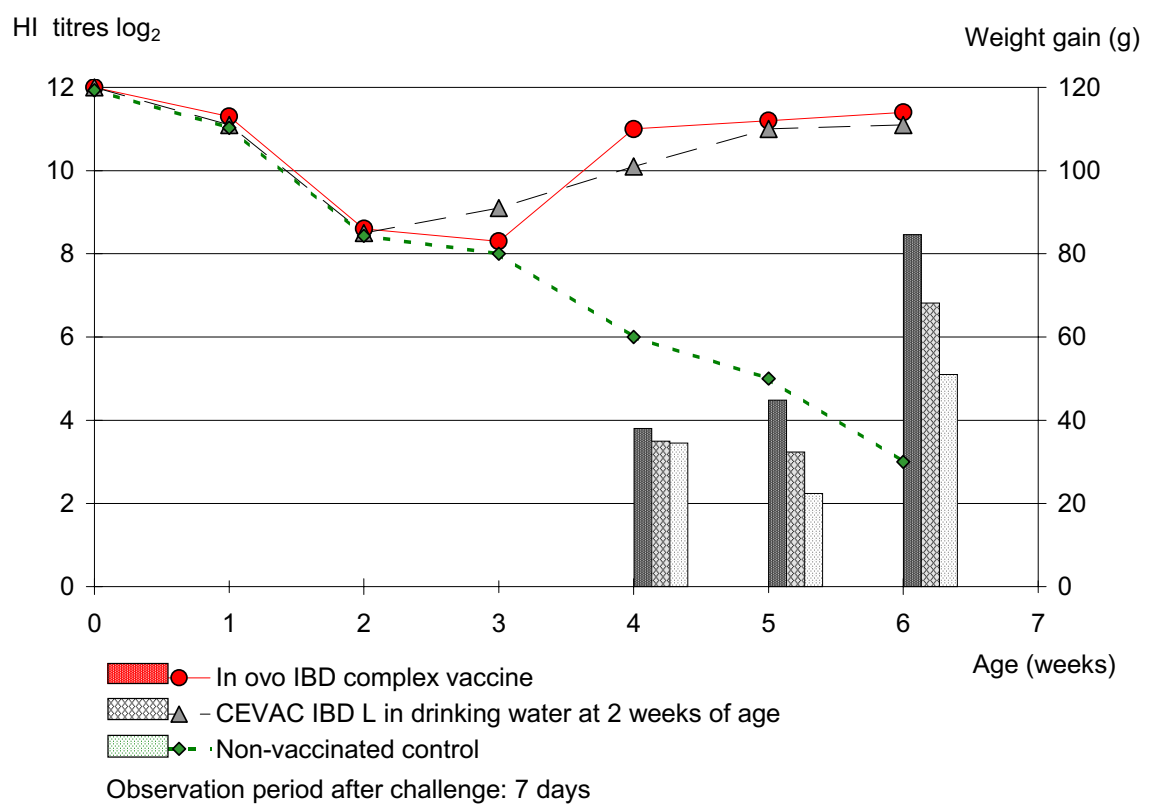

Fig. 4. Field trial: change of $\mathrm{VN}$ titres after vaccination and average daily weight gains after challenge at 4, 5 and 6 weeks of age

After chickens hatched from SPF embryos immunized with the complex vaccine in ovo were vaccinated with the Vitapest vaccine at day old, the VN titre of IBD antibodies was somewhat higher even than in the groups vaccinated with the IBD complex vaccine alone, and in weeks 3 and 5 the level of protection against IBD was $100 \%$ (Fig. 5). 


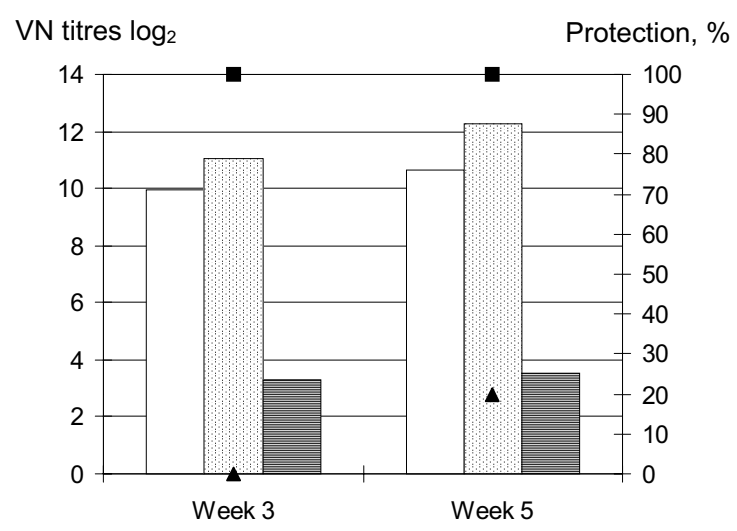

$\square$ In ovo VN
In ovo + Vitapest
晅 Control VN
$\square$ In ovo protection
$\Delta$ Control protection

Fig. 5. Protection and VN titre to infectious bursal disease in control SPF chickens and in chickens immunized with IBD complex vaccine in ovo and vaccinated with Vitapest at day old

In broiler chickens immunized in ovo, in weeks 3 and 5 after immunization with the Vitapest vaccine at day old the HI titres were on a level usually seen in flocks not immunized against IBD, and the immunized groups had $100 \%$ protection against Newcastle disease both in week 3 and in week 5 (Fig. 6).

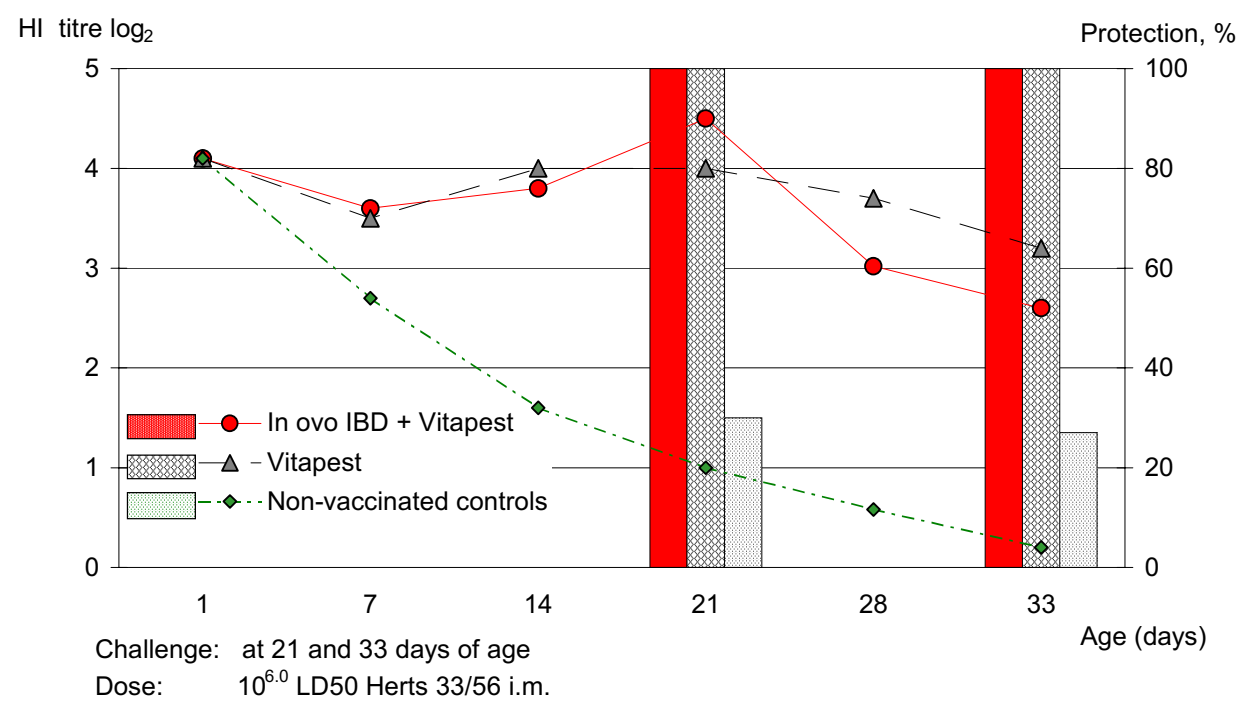

Fig. 6. Effect of in ovo IBD vaccination on the immune response to Vitapest vaccination performed at day old: protection and $\mathrm{HI}$ titre 
The anti-ND HI titres found in 3-week-old SPF and broiler chicken groups immunized in ovo with any of the 3 vaccine strains and with the complex vaccine and vaccinated at day old with the Vitapest vaccine clearly indicate that maternal antibodies to IBD or the antibodies contained in the complex vaccine markedly influence the immunosuppressive effect of the vaccines, reflected by the dissimilar HI titres. Although to a lesser extent, this also manifests itself in different levels of protection (Fig. 7).

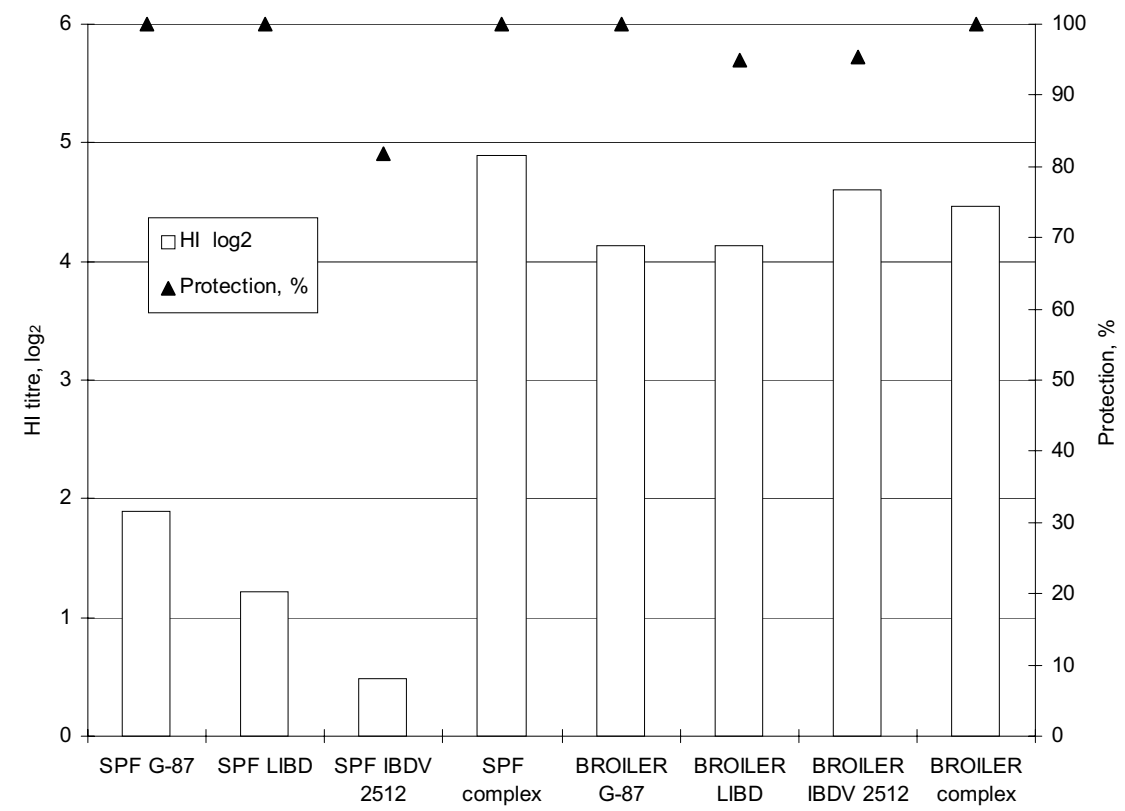

Fig. 7. Mean HI titre and protection against Newcastle disease on day 21 in chicken groups immunized with different IBD vaccines in ovo and vaccinated with Vitapest at day old

\section{Conclusions}

The comparative study of IBD vaccine virus strains to be used in the complex vaccine has shown both in SPF chicks and in broilers having maternal antibodies that the damage caused to the bursa of Fabricius increased in direct ratio to the virulence of the strain. The damage was more severe in SPF than in broiler embryos, and the most favourable values were obtained after the use of the complex vaccine. This histologically distinct lesion is of temporary nature (the damaged bursae will be restored to normal); however, in broilers and after the use of the complex vaccine this tissue repair is faster than after the vaccination of SPF embryos. This restoration of the bursa has been documented by Iván et al. (2000), who showed that in SPF chickens vaccinated in ovo depletion of B lym- 
phocytes of the bursa of Fabricius took place from day 7 after hatching, while in broilers this occurred much later, from 33 days of age onward. Bursal restoration lasts 3-4 days in SPF chickens and 11 days in broilers, after which time B lymphocytes repopulate the bursa of Fabricius. Kim et al. (1999) also found that the speed of bursal restoration depended on the virulence of the vaccine virus strain, and that after repair the production of antibodies to Newcastle disease commenced. Although the role of maternal antibodies or of antibodies contained by the complex vaccine in the prevention of immunosuppressive effect is not fully known, the virus present in the complex vaccine can be detected 5 days later than the vaccine virus included in a vaccine without antibody. The antibody present in the complex vaccine does not neutralize the virus but reduces its pathogenicity, resulting in less severe B lymphocyte depletion after the use of the complex vaccine (Haddad et al., 1997; Jeurissen et al., 1998).

Three weeks after day-old Vitapest vaccination of SPF chickens vaccinated against IBD in ovo the level of Newcastle disease antibodies detectable by the HI test decreased in inverse ratio to the virulence of the strain used for immunization against IBD. No such effect was observed after the in ovo immunization of broiler embryos. It is considered an important finding that 3 weeks after the day-old Vitapest vaccination of chickens hatched after in ovo vaccination with the complex vaccine the HI titre did not decrease in the SPF group either. It has also been clearly demonstrated that the above decline of the HI titre was in most cases not accompanied by the decrease of protection against Newcastle disease. This confirms the findings of other authors (Sharma and Lee, 1983; Craft et al., 1990; Kim et al., 1999), according to which IBD virus primarily inhibits humoral antibody synthesis. The mechanisms inhibiting $\mathrm{T}$ cell functions are yet insufficiently known (Sharma et al., 2000); however, in the present experiments cell-mediated protection decreased only slightly or not at all. Similar findings were reported by Giambrone (1979).

The experiments reported here have confirmed the previously reported correlation (Muskett et al., 1979; Nakamura et al., 1992) that the higher the virulence of the vaccine virus strain, the stronger the immune response but also the immunosuppressive effect. Of the 3 strains compared, the intermediate-plus strain IBDV 2512 administered in ovo could induce active antibody formation after the waning of the maternal antibodies. Other researchers have also found that the complex vaccine made from this strain, injected subcutaneously at day old or administered in ovo, is more effective than the commercial vaccine given in the drinking water (Haddad et al., 1997; Johnston et al., 1997; Jeurissen et al., 1998). According to our studies, the temporary immunosuppressive effect caused by strain IBDV 2512 was markedly diminished by the homologous antibody added to the strain. This was also indicated by the fact that the histologically detectable damage of the bursa of Fabricius persisted for the shortest time after the use of the complex vaccine. 


\section{References}

Craft, D.W., Brown, J. and Lukert, P. D. (1990): Effects of standard and variant strains of infectious bursal disease virus on infections of chickens. Am. J. Vet. Res. 51, 1192-1197.

Giambrone, J. J. (1979): Effects of early IBDV infection on immunity to Newcastle disease in adult chicken. Poultry Sci. 59, 794-798.

Haddad, E. E., Whitfill, C. E., Avakian, A. P., Riks, C. A., Andrews, P. D., Thoma, J. A. and Wakenell, P. S. (1997): Efficacy of a novel IBDV immune complex vaccine in broiler chickens. Avian Dis. 41, 882-889.

Iván, J., Nagy, N., Kacskovics, I. and Mészáros, J. (2000): Functional restoration of the bursa of Fabricius following infectious bursal disease vaccination. Vet. Immunol. Immunopathol. (submitted).

Jeurissen, S. H. M., Janse, E. M., Lehrbach, P. R., Haddad, E. E., Avakian, A. and Whitfill, C. E. (1998): The working mechanism of an immune complex vaccine that protect chickens against infectious bursal disease. Immunology 95, 494-500.

Johnston, P. A., Ziu, H., O’Conell, T., Phelps, P., Bland, M., Tyczkowski, J., Kemper, A., Harding, T., Avakian, A., Haddad, E., Whitfill, C., Gildersleeve, R. and Ricks, C. A. (1997): Applications in in ovo technology. Poultry Sci. 76, 165-178.

Kim, I. J., Gagic, M. and Sharma, J. M. (1999): Recovery of antibody-producing ability and lymphocyte repopulation of bursal follicles in chickens exposed to infectious bursal disease virus. Avian Dis. 43, 401-413.

Lütticken, D. (1997): Viral diseases of the immune system and strategies to control infectious bursal disease by vaccination. Acta Vet. Hung. 45, 933-938.

Mészáros, J., Szemerédi, M. and Tamási, G. (1992): Immunization of day-old chickens against Newcastle disease. Acta Vet. Hung. 40, 121-127.

Muskett, J. C., Hopkins, I. G., Edwards, K. R. and Thornton, D. H. (1979): Comparison of two vaccine strains: efficacy and potencial hazards in susceptible and maternally immune birds. Vet. Rec. 104, 332-334.

Nakamura, T., Otaki, Y. and Nunuya, T. (1992): Immunosuppressive effect of a highly virulent infectious bursal virus isolated in Japan. Avian Dis. 36, 891-896.

Sharma, J. M., Kim, I. J., Rautenschein, S. and Yeh, H. Y. (2000): Infectious bursal disease of chickens: pathogenesis and immunosuppression. Dev. Comp. Immunol. 24, 223-235.

Sharma, J. M. and Lee, L. F. (1983): Effect of infectious bursal disease on natural killer cell activity and mitogenic response of chicken lymphoid cells and the role of adherent cells in cellular immune suppression. Infect. Immun. 42, 747-754.

Süveges, T. (1998): Observations on the histopathology of alterations developed in the bursa of susceptible chickens due to virulent infectious bursitis virus (in Hungarian, with English abstract). Magy. Állatorv. Lapja 120, 73-79.

Van den Berg, T. P., Gonze, M. and Meulemans, G. (1991): Acute infectious bursal disease in poultry; isolation and characterization of highly virulent strains. Avian Pathol. 20, 133-143.

Whitfill, C. E., Haddad, E. E., Ricks, C. A., Skeeles, J. K., Newberry, L. A., Beasley, J. N., Andrews, P. D., Thoma, J. A. and Wakenell, P. S. (1995): Determination of optimum formulation of a novel infectious bursal disease virus (IBDV) vaccine constructed by mixing bursal antibody with IBDV. Avian Dis. 39, 687-699. 